\title{
Vitamin D Deficiency in End Stage Renal Disease Patients with Diabetes Mellitus Undergoing Hemodialysis
}

Muhammad Ali ${ }^{1}$, Ayesha Ejaz ${ }^{2}$, Shafique A. Solangi ${ }^{2}$, Abdul Manan Junejo ${ }^{2}$, Mahjabeen Yaseen ${ }^{1}$, Hina Iram $^{2}$, Sagheer Ahmed Solangi ${ }^{3}$

1. Nephrology, Fazaia Ruth Pfau Medical College, Karachi, PAK 2. Nephrology, Jinnah Postgraduate Medical Centre, Karachi, PAK 3. Medicine, Jinnah Postgraduate Medical Centre, Karachi, PAK

Corresponding author: Muhammad Ali, aliunar2002@yahoo.com

\section{Abstract}

Objectives: To assess the association of hypovitaminosis D with diabetes mellitus (DM) in patients with end stage renal disease (ESRD) undergoing hemodialysis.

Methodology: This cross-sectional study was conducted at the Jinnah Postgraduate Medical Centre between July 2019 and February 2020. Patients with diagnosed ESRD who were on hemodialysis, with or without concomitant DM were registered. Vitamin D levels were categorized according to the severity of the deficiency or excess as $0-10 \mathrm{ng} / \mathrm{mL}$, severely deficient; $11-20 \mathrm{ng} / \mathrm{mL}$, deficient; $21-32 \mathrm{ng} / \mathrm{mL}$; insufficient, $33-49 \mathrm{ng} / \mathrm{mL}$, adequate; $50-65 \mathrm{ng} / \mathrm{mL}$, optimum; and above that as high. Patients were stratified according to the status of DM.

Results: In a total of 80 , the mean age was $45.21 \pm 12.67$ years with 51 (63.75\%) males and 29 (36.25\%) females. A total of 36 (45\%) CKD patients had concomitant diabetes. The median vitamin D levels were $20.25 \mathrm{ng} / \mathrm{mL}$. It was found that chronic kidney disease (CKD) patients with concomitant DM had significantly lower levels of vitamin D [15.19 \pm 6.83 vs. $30.28 \pm 14.22$ ( $\mathrm{p}<0.001)]$. Out of the 12 patients with a severe deficiency, three-fourths of the population had DM as comorbidity, while in those with 'deficiency', 19 (67.9\%) had DM. The majority of the patients without DM had adequate or optimum levels of serum 25hydroxyvitamin D levels.

Conclusion: Current study indicated that deficiency of serum vitamin D is associated with concomitant DM in patients with CKD as the majority had a severe deficiency of serum 25(OH)D. Supplemental vitamin D may help correct the deficiency and prevent the associated complications in patients.

Categories: Endocrinology/Diabetes/Metabolism, Internal Medicine, Nephrology

Keywords: chronic kidney disease, diabetes mellitus type 2, hypovitaminosis, vitamin d

Review began 10/31/2020 Review ended 11/23/2020 Published 11/23/2020

\section{() Copyright 2020}

Ali et al. This is an open access article distributed under the terms of the Creative Commons Attribution License CC-BY 4.0., which permits unrestricted use, distribution, and reproduction in any medium, provided the original author and source are credited.

\section{Introduction}

Vitamin D is a fat-soluble vitamin that is produced endogenously in the skin depending on the ultraviolet rays from the sun, which when in contact with the skin triggers vitamin D synthesis in the body. For most individuals, around $90 \%$ of vitamin D is produced in this way, whereas the remaining $10 \%$ is obtained from food and dietary supplements [1-2].

Previously, it was known that this vitamin only plays a role in the regulation of calcium and phosphate in our body [2]. However, more recently low levels of vitamin D have also been linked with many other conditions including bone diseases, cardiovascular diseases, and many psychiatric ailments [3-5].

Provitamin from the skin or diet after hydroxylation in the liver forms 25 -hydroxyvitamin D [25(OH)D]. It is converted into its activated form (1,25-dihydroxyvitamin D) in the kidneys [6]. In chronic kidney disease (CKD) patients, this final step of active vitamin D production is impaired. Moreover, the hyperphosphatemic-osteocyte-derived hormone, i.e. fibroblast growth factor (FGF-23) increases to compensate for phosphate retention and further inhibits the renal $1 \alpha$-hydroxylase expression, inducing the expression of 24-hydroxylase responsible for the degradation of 1,25-dihydroxyvitamin D3. However, 24,25dihydroxyvitamin D3 levels are lower in dialysis patients than in the normal population. Thus, the impaired uptake of 25-hydroxyvitamin D3 by altered kidneys remains the main cause of 1,25-dihydroxyvitamin D3 deficiency [7].

Another important clinically significant discussion could be of the substrate deficit, i.e. decreased 25(OH)D. Patients with CKD or those on hemodialysis are believed to have reduced cutaneous synthesis than normal individuals as well as increased melanin pigmentation, even if the sunlight exposure is identical. This might be the reason for the low levels of 25(OH)D. Other reasons may be inactivity (low exposure) or inadequate 
calcium containing diet. Interestingly, they have a direct relationship between 1,25 dihydroxyvitamin $\mathrm{D}$ and $25(\mathrm{OH}) \mathrm{D}$ unlike normal individuals, the exact cause of which is unknown $[8,9]$.

Evidence has shown that increasing age, female gender, proteinuria, physical inactivity, diabetes mellitus $(\mathrm{DM})$, and nutritional deficiencies are correlated with hypovitaminosis D in patients with CKD $[5,6]$. Nephropathy is a serious complication that develops in patients with DM. Diabetic kidney disease affects about one-third of patients with DM and currently ranks as the foremost cause of end-stage renal disease (ESRD) [10]. Low levels of vitamin D have been reported in CKD patients with concomitant DM undergoing hemodialysis therapy [5]. Furthermore, Drechsler et al. reported that deficiency of vitamin D in patients on hemodialysis leads to adverse outcomes and is associated with high mortality among these patients [6].

Despite a high prevalence of vitamin D deficiency as well as DM, especially in Pakistan the local data has been very limited $[11,12]$. Hence, the current study was undertaken to fill the gap in the local literature. The current study aimed to determine the association between vitamin D deficiencies in CKD patients with DM undergoing hemodialysis compared to non-diabetic patients in our setting.

\section{Materials And Methods}

This cross-sectional observational study was conducted at the Nephrology Department, Jinnah Postgraduate Medical Centre, Karachi, Pakistan between July 2019 and February 2020. Ethical approval was procured from its Institutional Review Board. A non-probability convenience sampling technique was applied and 80 patients with diagnosed ESRD who were on hemodialysis, with or without concomitant DM were registered in the specified duration. All patients were included in the study after informed verbal and written consent.

According to the World Health Organization, vitamin D deficiency is defined as the serum 25(OH) D levels of less than $20 \mathrm{ng} / \mathrm{mL}$ [13]. For this study, vitamin D levels were categorized according to the severity of the deficiency or excess among the participants. It was grouped into seven classes as: severely deficient 0$10 \mathrm{ng} / \mathrm{mL}$, deficient $11-20 \mathrm{ng} / \mathrm{mL}$, and insufficient $21-32 \mathrm{ng} / \mathrm{mL}$, adequate $33-49 \mathrm{ng} / \mathrm{mL}$, optimum 50-60 $\mathrm{ng} / \mathrm{mL}$, toxic $60-70 \mathrm{ng} / \mathrm{mL}$ and above that as potentially toxic [14].

For blood samples, an experienced nurse was appointed who had experience of more than three years. A 5 $\mathrm{ml}$ blood sample was collected from each patient, under aseptic conditions using a tourniquet. Patients' vitals were monitored regularly. Serum samples were collected for hemoglobin $(\mathrm{Hb})$ levels, serum vitamin $\mathrm{D}$, intact parathyroid hormone (iPTH), urea and creatinine, hemoglobin A1c (HbA1c), ferritin, transferrin, random blood sugar (RBS), and fasting blood sugar (FBS). Patients were stratified according to the status of DM diagnosed by RBS, FBS, and HbA1c. For all participants, demographic data and clinical characteristics were entered into a predefined questionnaire.

Data were analyzed using IBM Statistical Package for the Social Sciences (SPSS) Statistics for Windows, version 21.0. (IBM Corp., Armonk, NY, US). The average age, Hb, vitamin D, iPTH, among other continuous variables were presented as mean and standard deviation. For vitamin D levels, the median was also presented. Categorical data were represented as frequency and percentages. Association of vitamin D levels with DM status was observed using a Chi-square test and an independent $\mathrm{t}$-test. A $\mathrm{p}$-value $<0.05$ was set as the cut-off value for significance.

\section{Results}

In a total of 80 participants, the mean age was $45.21 \pm 12.67$ years with a range of $18-40$ years. There were 51 (63.75\%) male and 29 (36.25\%) female participants. The mean values for over all serum $\mathrm{Hb}$, iPTH, vitamin D levels, calcium and phosphate are given in Table 1 . 


\section{Cureus}

\section{Variable}

Age $\pm S D$ in years

Hemoglobin \pm SD in $\mathrm{mg} / \mathrm{dL}$

$\mathrm{iPTH} \pm \mathrm{SD}$ in pg/dL

Vitamin $D \pm S D$ in $\mathrm{ng} / \mathrm{dL}$

Serum ferritin $\pm S D$ in $\mathrm{pg} / \mathrm{dL}$

Iron $\pm \mathrm{SD}$ in $\mathrm{mcg} / \mathrm{dL}$

Calcium \pm SD in $\mathrm{mg} / \mathrm{dL}$

Phosphate \pm SD in $\mathrm{mg} / \mathrm{dL}$

Albumin \pm SD in $\mathrm{mg} / \mathrm{dL}$

$\mathrm{URR} \pm \mathrm{SD}$ in percent

SpKt/V

Dialysis Duration \pm years

Dialysis sessions-week
Mean \pm Standard Deviation

$45.21 \pm 12.67$

$9.81 \pm 1.7$

$592.4 \pm 624.78$

$23.5 \pm 13.70$

$560.27 \pm 371.49$

$91.50 \pm 41.61$

$8.11 \pm 0.824$

$5.673 \pm 1.705$

$3.33 \pm 0.538$

$64.074 \pm 4.404$

$1.225 \pm 0.115$

$5.212 \pm 1.004$

$2.606 \pm 0.5126$

\section{TABLE 1: Clinical Characteristic of Patients in the Study Population}

Abbreviations: SD: Standard deviation; URR: Urea reduction ratio; SpKt/V: Single pool Kt/V where Kt/V shows dialysis adequacy by incorporating dialyzer clearance of urea $(\mathrm{K})$, dialysis time $(\mathrm{t})$, and volume of distribution of urea (V); iPTH: Intact Parathyroid hormone

We have stratified patients, a total of 36 (45\%) ESRD patients had concomitant diabetes while 44 (55\%) did not have diabetes as a comorbidity. The overall mean vitamin D levels were $23.5 \pm 13.70$ with a range of 4.82$68.90 \mathrm{ng} / \mathrm{dL}$. It was found that ESRD patients with concomitant DM had significantly lower levels of vitamin D compared to patients without diabetes as comorbidity [20.517 \pm 12.447 vs. $30.021 \pm 12.810$ (p<0.0004)]. Out of the 12 patients with a severe deficiency, three-fourths of the population had DM as comorbidity. Similarly, out of the 28 patients with deficient levels, 19 (67.9\%) had DM (Table 2). 


\section{Cureus}

\begin{tabular}{|c|c|c|c|}
\hline$n=94$ & & Diabetic $n=45$ & Non Diabetic $n=49$ \\
\hline Variable & $P=$ value & Mean \pm Standard Deviation & Mean \pm Standard Deviation \\
\hline Age & 0.577 & $46.31 \pm 13.534$ & $44.78 \pm 13.039$ \\
\hline Uric Acid & 0.222 & $6.980 \pm 2.069$ & $6.3790 \pm 1.909$ \\
\hline Hemoglobin & 0.861 & $9.2342 \pm 1.329$ & $9.285 \pm 1.516$ \\
\hline PTH & 0.721 & $563.133 \pm 580.703$ & $606.101 \pm 585.320$ \\
\hline Iron & 0.948 & $93.952 \pm 49.391$ & $93.343 \pm 39.910$ \\
\hline Feritin & 0.242 & $641.2267 \pm 429.775$ & $548.574 \pm 368.994$ \\
\hline Calcium & 0.755 & $8.1398 \pm 0.855$ & $8.0861 \pm 0.802$ \\
\hline Phosphate & 0.437 & $5.687 \pm 1.477$ & $5.6602 \pm 1.906$ \\
\hline Albumin & 0.885 & $3.347 \pm 0.495$ & $3.3310 \pm 0.580$ \\
\hline URR & 0.753 & $64.93 \pm 4.002$ & $64.67 \pm 3.996$ \\
\hline SpKt/V & 0.816 & $1.1489 \pm 0.132$ & $1.1429 \pm 0.117$ \\
\hline Year & 0.045 & $4.422 \pm 0.811$ & $4.326 \pm 0.875$ \\
\hline Sessions $/ W$ & 0.918 & $2.622 \pm 0.490$ & $2.632 \pm 0.487$ \\
\hline Vitamin D & 0.0004 & $20.517 \pm 12.447$ & $30.021 \pm 12.810$ \\
\hline
\end{tabular}

\section{TABLE 2: Comparison of parameters between diabetic and non-diabetic patients}

Abbreviations: URR: Urea reduction ratio; SpKt/V: Single pool Kt/V where Kt/V shows dialysis adequacy by incorporating dialyzer clearance of urea $(\mathrm{K})$, dialysis time $(\mathrm{t})$, and volume of distribution of urea (V); PTH: Parathyroid hormone

In our study adequacy of hemodialysis was also assessed by urea reduction ratio and single pool Kt/V; in the diabetic group mean URR was $64.93 \pm 4.002$ while in the non-diabetic group it was $64.67 \pm 3.996$ with a Pvalue of 0.753

$\mathrm{SpKt} / \mathrm{V}$ was $1.1489 \pm 0.132$ in the diabetic group and in the non-diabetic group it was $1.1429 \pm 0.117$ (P-value $0.816)$.

Mean duration of hemodialysis patients was $5.212 \pm 1.004$ years, while in the diabetic group duration of hemodialysis $4.422 \pm 0.811$ years and in the non-diabetic group it was noted $4.326 \pm 0.875$ with significant P-value (0.045).

The majority of patients without diabetes mellitus had adequate or optimum levels of serum 25hydroxyvitamin D levels (Figure 1).

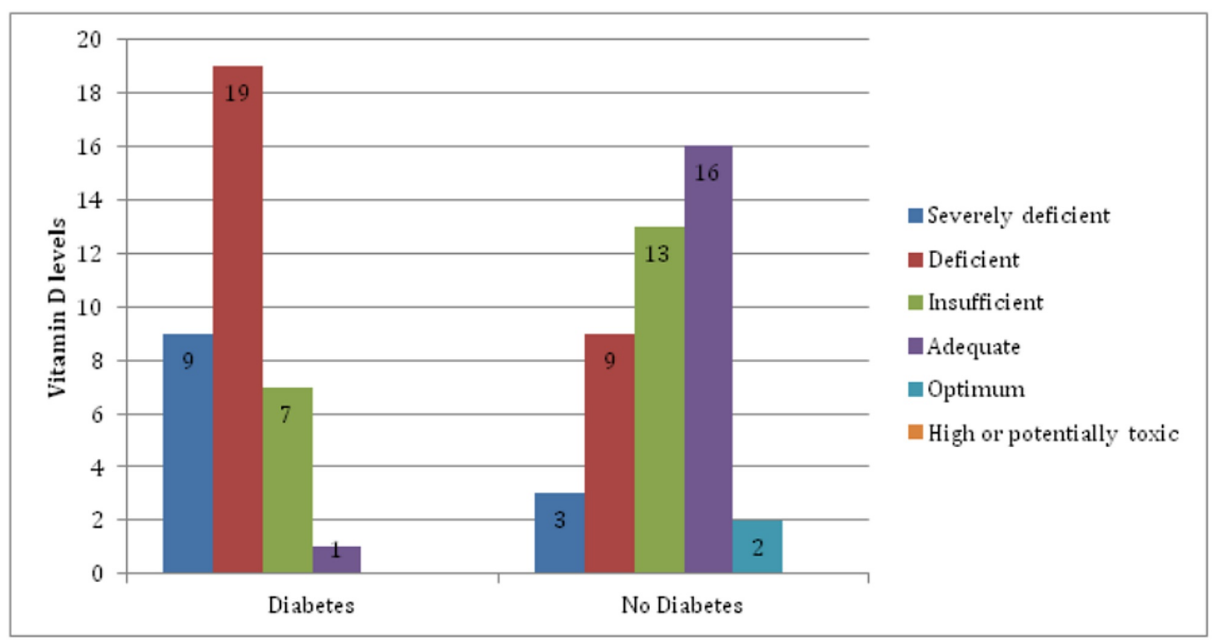




\section{Discussion}

The degree of association of vitamin $\mathrm{D}$, serum mineral regulation, and the physiological function of the renal system necessitate a vast body of research that may provide relevant clinical insight. In this study, the mean values for $\mathrm{Hb}$, $\mathrm{iPTH}$, and vitamin $\mathrm{D}$ were decreased, increased, and normal respectively following standard ranges. These findings demonstrate that overall vitamin $\mathrm{D}$ deficiency was not particularly prevalent in this population of ESRD patients; although these levels did not reflect a deficiency, they are still relatively low within the normal range.

Even though vitamin D deficiency was not demonstrated based on mean serum levels within the population, this study was able to find a statistically significant difference between vitamin D serum levels relative to non-diabetic ESRD patients. As stated earlier in the results, out of the patients with the greatest level of vitamin D deficiency, the vast majority of them had DM as associated comorbidity. Other studies have demonstrated a linear progressive relationship between diabetic nephropathy patients and vitamin D deficiency [15]. Sacerdote et al. performed a focused review and found an inverse relationship between vitamin D levels and type 2 diabetes, as well as other insulin-related disorders [16].

The causal mechanism involved in the onset and progression of diabetes in association with vitamin D likely concerns the role of the vitamin in the immune system and insulin secretion. For instance, vitamin D serves an important immunomodulatory function, and its respective receptor (VDR) is found on both $\mathrm{T}$ and $\mathrm{B}$ lymphocytes. The modification of the Thelper cell cytokine profile can induce inhibition and expression of effectors T cells, such as those involved in autoimmune reactions which may lead to type $1 \mathrm{DM}$, vitamin D deficiency also inhibits pancreatic insulin secretion from the beta islet cells, contributing to complications observed in type 2 diabetes [17]. The clinical implications of these findings may be especially immense given local studies that found statistical significance among vitamin-deficient patients and elevated HbA1C levels, blood glucose, and poor glycemic control [18]. Mahmood et al. concluded that measures should be taken to avoid unfavorable clinical outcomes in DM patients through either vitamin supplementation or increased exposure to sunlight [19].

Despite efforts to generalize the findings, this study still posed several limitations such as limited sample size and a primarily older, male, and anemic demographic. The clinical profile and demographics of the patients provide preconditions that are inextricably linked to the propensity for hypovitaminosis. It is well known that the process of aging affects the formation of vitamin D. In fact, an approximately $50 \%$ reduction of production is observed due to a decline in renal function which is largely caused by progressing age. The development of a deficiency leads to a greater reduction in the formation of the metabolite, initiating a sort of positive feedback loop. Besides, gender has also been found to play a largely significant role in vitamin D status. In a study evaluating patients undergoing coronary angiography, females had higher rates of renal failure and were associated with lower vitamin D levels by a large statistical margin. Furthermore, recent studies have found that vitamin D deficiency tends to coincide with anemia in both healthy and diseased populations, and may even be involved in the causal mechanism of its development. Granted its involvement in erythropoiesis and the suppression of hepcidin, there is evidence that sufficient supplementation of the vitamin may be an effective preventative measure against the development of anemia [20-22].

There is significant relationship between duration of hemodialysis and vitamin $\mathrm{D}$ levels in diabetic patients on hemodialysis (0.045), while Bansal et al. in 2012 from India found weak correlation between duration and vitamin D [23]. El-Arbagy et al. from Egypt recently stated that there is no correlation between duration and vitamin D level [24].

\section{Conclusions}

The current study indicated that deficiency of serum vitamin D is associated with concomitant DM in patients with ESRD as the majority had a severe deficiency of serum 25(OH)D. Supplemental vitamin D may help correct the deficiency and prevent the associated complications in patients.

\section{Additional Information \\ Disclosures}

Human subjects: Consent was obtained by all participants in this study. Jinnah Postgraduate Medical Centre issued approval N0.F.2-81-IRB/2020-GEN/42514/JPMC. With reference to your application/letter dated 20th February, 2020, on the subject noted above and to say that institutional review board has allowed to retrieve data. Animal subjects: All authors have confirmed that this study did not involve animal subjects or tissue. Conflicts of interest: In compliance with the ICMJE uniform disclosure form, all authors 
declare the following: Payment/services info: All authors have declared that no financial support was received from any organization for the submitted work. Financial relationships: All authors have declared that they have no financial relationships at present or within the previous three years with any organizations that might have an interest in the submitted work. Other relationships: All authors have declared that there are no other relationships or activities that could appear to have influenced the submitted work.

\section{References}

1. Göring H: Vitamin D in nature: a product of synthesis and/or degradation of cell membrane components . Biochem. 2018, 83:1350-7. 10.1134/S0006297918110056

2. DeLuca HF: Vitamin D: historical overview. Vitam Horm. 2016, 100:1-20. 10.1016/bs.vh.2015.11.001

3. Drechsler C, Pilz S, Obermayer-Pietsch B, Verduijn M, Tomaschitz A, Krane V: Vitamin D deficiency is associated with sudden cardiac death, combined cardiovascular events, and mortality in haemodialysis patients. Eur Heart J. 2010, 31:2253-61. 10.1093/eurheartj/ehq246

4. Heath AK, Kim IY, Hodge AM: Vitamin D status and mortality: a systematic review of observational studies . Int J Environ Res Public Health. 2019, 16:383. 10.3390/ijerph16030383

5. Jean G, Souberbielle JC, Chazot C: Vitamin d in chronic kidney disease and dialysis patients . Nutrients. 2017, 9:328. 10.3390/nu9040328

6. Drechsler C, Verduijn M, Pilz S, et al.: Vitamin D status and clinical outcomes in incident dialysis patients: results from the NECOSAD study. Nephrol Dial Transplant. 2011, 26:1024-32. 10.1093/ndt/gfq606

7. Quarles LD: Role of FGF23 in vitamin D and phosphate metabolism: implications in chronic kidney disease . Exp Cell Res. 2012, 318:1040-8. 10.1016/j.yexcr.2012.02.027

8. Al-Badr W, Martin KJ: Vitamin D and kidney disease . Clin J Am Soc Nephrol. 2008, 3:1555-60. 10.2215/CJN.01150308

9. Restrepo Valencia CA, Aguirre Arango JV: Vitamin D (25(OH)D) in patients with chronic kidney disease stages 2-5. Colomb Medica (Cali). 2016, 47:160-6. 10.25100/cm.v50i1.4444

10. John S: Complication in diabetic nephropathy. Diabetes Metab Syndr. 2016, 10:247-9. 10.1016/j.dsx.2016.06.005

11. Riaz H, Finlayson AE, Bashir S, Hussain S, Mahmood S, Malik F, Godman B: Prevalence of vitamin D deficiency in Pakistan and implications for the future. Expert Rev Clin Pharmacol. 2016, 9:329-38. 10.1586/17512433.2016.1122519

12. Akhtar S, Nasir JA, Abbas T, Sarwar A: Prevalence of vitamin D deficiency in Pakistan and implications for the future. Pak J Med Sci. 2019, 35:1173-8. 10.12669/pjms.35.4.194

13. Prevention and management of osteoporosis: report of a WHO scientific group . (2003). Accessed: August 23, 2020: https://apps.who.int/iris/handle/10665/42841.

14. Grundmann M, von Versen-Höynck F: Vitamin D - roles in women's reproductive health? . Reprod Biol Endocrinol. 2011, 9:146. 10.1186/1477-7827-9-146

15. Xiao X, Wang Y, Hou Y, Han F, Ren J, Hu Z: Vitamin D deficiency and related risk factors in patients with diabetic nephropathy. J Int Med Res. 2016, 44:673-84. 10.1177/0300060515593765

16. Sacerdote A, Dave P, Lokshin V, Bahtiyar G: Type 2 diabetes mellitus, insulin resistance, and vitamin D . Curr Diab Rep. 2019, 19:101. 10.1007/s11892-019-1201-y

17. Nakashima A, Yokoyama K, Yokoo T, Urashima M: Role of vitamin D in diabetes mellitus and chronic kidney disease. World J Diabetes. 2016, 7:89-100. 10.4239/wjd.v7.i5.89

18. Iqbal K, Islam N, Mehboobali N, Asghar A, Iqbal MP: Association of vitamin D deficiency with poor glycaemic control in diabetic patients. J Pak Med Assoc. 2016, 66:1562-5.

19. Mahmood Y, Shahid S, Fawad A, Basit A, Azhar A: Association of vitamin D with type 2 diabetes mellitus in Karachi, Pakistan. Int J Biol Biotechnol. 2018, 15:201-5.

20. Gallagher JC: Vitamin D and aging. Endocrinol Metab Clin North Am. 2013, 42:319-32. 10.1016/j.ecl.2013.02.004

21. Verdoia M, Schaffer A, Barbieri L, Di Giovine G, Marino P, Suryapranata H: Impact of gender difference on vitamin D status and its relationship with the extent of coronary artery disease. Nutr Metab Cardiovasc Dis. 2015, 25:464-70. 10.1016/j.numecd.2015.01.009

22. Smith EM, Tangpricha V: Vitamin D and anemia: insights into an emerging association . Curr Opin Endocrinol Diabetes Obes. 2015, 22:432-8. 10.1097/MED.0000000000000199

23. Bansal B, Bansal S, Mithal A, Kher V, Marwaha R: Vitamin D deficiency in hemodialysis patients . Indian J Endocr Metab. 2012, 16:270-3. 10.4103/2230-8210.93749

24. El-Arbagy AR, El-Zorkany KM, Helwa MA, El-Khalifa EA: Assessment of vitamin D in hemodialysis patients . Menoufia Med J. 2020, 33:122-6. 10.4103/mmj.mmj_317_18 